\title{
1000 human genomes carry widespread signatures of GC biased gene conversion
}

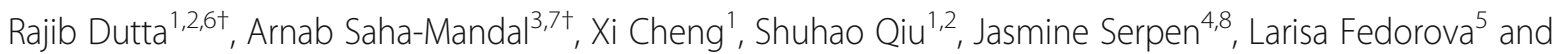
Alexei Fedorov $2,3^{*}$

\begin{abstract}
Background: GC-Biased Gene Conversion (gBGC) is one of the important theories put forward to explain profound long-range non-randomness in nucleotide compositions along mammalian chromosomes. Nucleotide changes due to $\mathrm{gBGC}$ are hard to distinguish from regular mutations. Here, we present an algorithm for analysis of millions of known SNPs that detects a subset of so-called "SNP flip-over" events representing recent gBGC nucleotide changes, which occurred in previous generations via non-crossover meiotic recombination.

Results: This algorithm has been applied in a large-scale analysis of 1092 sequenced human genomes. Altogether, 56,328 regions on all autosomes have been examined, which revealed 223,955 putative gBGC cases leading to SNP flip-overs. We detected a strong bias $(11.7 \% \pm 0.2 \%$ excess) in AT- > GC over GC- > AT base pair changes within the entire set of putative gBGC cases.

Conclusions: On average, a human gamete acquires 7 SNP flip-over events, in which one allele is replaced by its complementary allele during the process of meiotic non-crossover recombination. In each meiosis event, on average, gBGC results in replacement of 7 AT base pairs by GC base pairs, while only 6 GC pairs are replaced by AT pairs. Therefore, every human gamete is enriched by one GC pair. Happening over millions of years of evolution, this bias may be a noticeable force in changing the nucleotide composition landscape along chromosomes.
\end{abstract}

Keywords: SNP, Polymorphism, Bioinformatics, Mutation, Evolution

\section{Background}

One of the longstanding questions in mammalian genome evolution has been the origin of GC-isochors, which are long (> $100 \mathrm{~kb})$ chromosome segments characterized by a high degree of uniformity in GC-composition levels $[1,2]$. Several theories including selectionism [3], neutralism [4], thermodynamic stability [5], and GC-Biased Gene Conversion (gBGC) [6] have been proposed to explain the origin of isochors, but have not been conclusive. The gBGC hypothesis was initially formulated by Holmquist [7] and Eyre-Walker [8, 9], and has been elaborated upon since then $[10,11]$. gBGC is proposed to be a consequence of special cases of meiotic recombinations that involve the

\footnotetext{
* Correspondence: Alexei.fedorov@utoledo.edu

${ }^{\dagger}$ Equal contributors

${ }^{2}$ Department of Medicine, University of Toledo, Health Science Campus, Toledo, OH 43614, USA

${ }^{3}$ Program in Bioinformatics and Proteomics/Genomics, University of Toledo, Health Science Campus, Toledo, OH 43614, USA

Full list of author information is available at the end of the article
}

formation of heteroduplexes [11, 12]. A heteroduplex is created when a short single-stranded DNA segment of one of the parental chromosomes forms a double stranded structure with its complementary homologous strand from the equivalent chromosome of another parent. The presence of SNPs within a heteroduplex results in mismatched base pairs that are resolved by the molecular machinery of the DNA mismatch repair (MMR) pathway [13]. gBGC hypothesizes a bias in the repair, in which mismatched nucleotide pairs are resolved in favor of G-C pairs [10]. This would imply that mismatches involving non-Watson-Crick base pairs such as A-G, T-C, A-C or T-G would be preferentially repaired to yield C-G, G-C, G-C and C-G Watson-Crick base pairs respectively. In 2013, Lesecque and co-authors experimentally confirmed the existence of such bias in yeast, yet it appeared to be very small $(50.6 \%$ vs $49.4 \%$ in AT - > GC base pair changes vs. GC - > AT ones) [14].

One important consequence of gBGC is the increased overall GC-content at recombination hotspots [15], where 
heteroduplexes are more frequent than elsewhere. There are both supporting and opposing evidences for gBGC. The supporting propositions posit that gBGC explains the evolution of non-randomness in GC-compositions within mammalian genomes [11], the rapid fixation of AT- $>$ GC mutations $[11,16]$ and increased GC content of recombining DNA in mammals and yeast [17]. Evidence of gBGC has also been reported in yeasts [18], arabidopsis [19], and honeybee genomes [20]. On the other hand, conflicts with the gBGC hypothesis have also been published. For example, analysis of GC/AT and AT/GC substitutions in the human Fetuin-A gene ruled out gBGC as one of the causal factors [21]. Population genomic analysis of Drosophila melanogaster revealed no evidence for gBGC [22] and, in another instance, non-allelic gene conversion processes in Drosophila and primate genomes also negated the contribution of gBGC towards organism diversity [23]. A negative correlation between substitution and recombination rates in the chicken genome was also reported, inconsistent with the gBGC model [24]. Even though gBGC with respect to humans has been reported in recombination hotspots and rapidly-evolving regions of the human genome [25, 26], a quantitative picture of gBGC inside any region of the human genome was lacking until recently [11]. In the last 2 years, new publications reported directly observed cases of gBGC events in several large families [27, 28]. These authors demonstrated a strong bias in AT- > GC over GC-> AT base pair changes during non-crossover gene conversions in humans.

In the modern time when whole-genome sequencing is a routine and thousands of human genomes are available, is it possible to detect and quantify gBGC events in a large scale? One of the fundamental principles in genome organization is that neighboring SNPs are linked into haplotypes. A gene conversion event results in replacement of an allele inherited from one parent by the complementary allele inherited from another parent. About half of gBGC episodes occur during crossover $(\mathrm{CO})$ miotic recombinations while another half during non-crossover (NCO) recombinations. In the latter case of $\mathrm{NCO}$, the gBGC allele replacement occurs within the same parental haplotype producing only single nucleotide change inside this haplotype. We call this specific process a "SNP flip-over" throughout this paper. In other words, SNP flip-over is an allele replacement event for a single SNP in the middle of evolutionarily conserved haplotype. Such SNP flip-over may be directly detected by comparing the genomes of mother, father, and offspring, when haplotypes of all three are available. However, since the New Generation Sequencing technique is dependent on assembling millions of short reads, haplotypes are computationally deduced in a so-called "phasing" procedure. It is a statistical prediction that generates a vast number of phasing errors [29-31]. Phasing problems make the detection of de novo gBGC events difficult. To overcome this problem Williams with coauthors and Halldorsson with coauthors studied threegeneration pedigrees with multiple family members [27, 28]. Such direct detection of gBGC is of ultimate importance for the validation of this phenomenon yet it provides limited statistics.

On the other hand, Glemin and co-authors used analysis of derived allele frequency from the 1000 Genomes data to quantify gBGC in human [32]. We chose another approach for detection of relatively recent SNP flip-overs, due to gBGC that happened hundreds or thousands years ago. We computationally analyzed only common haplotypes built from frequent SNPs that remained unchanged in different populations for thousands of years. Then, we looked for a very rare haplotype that is practically identical to one of the common haplotypes except with one allele replacement (flip-over) at one of the polymorphic sites in the middle of the haplotype. We called this specific type of rare haplotype as "Acceptor" haplotype (See Fig. 1). We searched 1092 sequenced genomes for people that have the acceptor haplotype from one parent and its nearly identical common haplotype counterpart inherited from another parent (as illustrated in Fig. 1b). This requirement for parental haplotype organization is important for avoiding possible errors due to low sequencing coverage in the 1000 Genomes dataset. Indeed, under such conditions all polymorphic sites within a haplotype under analysis are homozygous except the one "Acceptor" site representing putative gBGC conversion event. This homozygosity requirement eliminates possible phasing errors.

In this paper, we performed bioinformatics investigation of SNPs in the "1000 Genomes" database and observed 223,955 putative cases of gBGC events. We report here a quantitative assessment of the aftermath of gBGC in.

\section{Methods}

Genotype datasets for all the human chromosomes of the 1092 human genomes were downloaded from the 1000 Genomes $\mathrm{ftp}$ site (ftp://ftp-trace.ncbi.nih.gov/ 1000genomes/ftp/release/20110521/) [33] as Variant Call Format (VCF) files version 4.1 [34]. This database contains a total of 38.2 M SNPs, 3.9 M short indels and $14 \mathrm{~K}$ deletions for all the human chromosomes that have been used in this study. Information about parental haplotypes has been taken directly from Phase 1 of 1000 Genomes Project, since its genomic sequences were entirely "phased". We considered only bi-allelic variants for simplicity and because we had sufficient statistics in our datasets. All multi-allelic variants were skipped. Allele frequency for every SNP was obtained from the "AF $=$ " field inside column 8 of the 1000 Genome VCF 
a Arrangement of segments and haplotypes in Chromosome 1

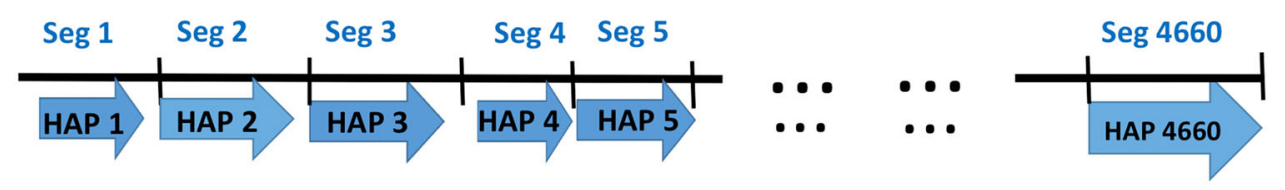

b

Chromosome 1 Segment 23

COMMON HAPLOTYPES OCCURRENCE

$11101110101101101100111111111111010111001111111110 \Rightarrow 216$
$11101111101101101100111111111111010111001111111110 \Rightarrow 168$
$00010000010010010011000000000000101000110000000001 \Rightarrow 103$

Haplotypes of Individual TSI NA20787:

Parent_1 $\quad 1110111010110 \overline{1} 10110011111111111101011100111111110$

Parent_2 111011101011011011001110111111101011100111111110
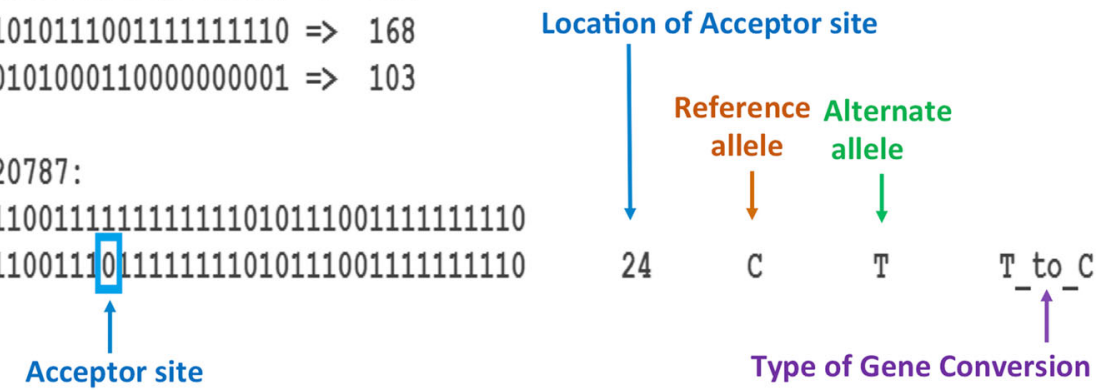

Fig. 1 Characterization of haplotypes of frequent genetic variants and putative case of BGC event. $\mathbf{a}$ - arrangement of computationally processed chromosomal segments for analysis of haplotypes. Autosomes have been divided into 56,328 segments, each containing 50 high-frequency (MAF > 25\%) genetic variants. b An example of common haplotypes inside the segment 23 of Chromosome 1. Haplotypes were constructed from 50 adjacent high-frequency (MAF > 25\%) genetic variants and are represented by the strings of fifty $0 \mathrm{~s}$ and $1 \mathrm{~s}$, where " 0 " means the presence of a reference allele, while "1" means an alternative allele in the haplotype. The haplotypes that occur $\geq 100$ times in the 1092 individuals are defined as 'common haplotypes' and are listed in descending order of their occurrence. The exemplified segment 23 has three common haplotypes. Putative BGC events were searched only in individuals who have one common haplotype and another nearly identical rare haplotype, which has only one allele difference with the common haplotype at the "acceptor" site (marked with a blue square). In this example such conditions were found in individual NA20787 from the TSI population. In the two parental haplotypes of TSI_NA20787, the first (Parent 1) is a common haplotype, which occurs 216 times in the 1092 genomes. The other haplotype (Parent 2), despite being identical to the common haplotype at 49 polymorphic sites, is a rare haplotype which occurs only once in the 1092 individuals. This rare haplotype contains the Acceptor site (marked with a blue square), which represents a case of putative base pair conversion event at this location in one of the ancestors of this individual. The location of this acceptor site in the haplotype string, its reference and alternative alleles, and base change due to BGC event (purple arrow) is shown in the bottom of the figure. Detailed information about every segment and all putative BGC events are available from our web site

files. We did not take into account differences in population frequencies in our paper because this frequency variation is not relevant at all for finding SNP flip-over events, which is the major focus in this study.

All haplotypes of 1092 individuals, putative cases of gene conversion and local GC content of putative gene conversion sites were obtained with our pipeline of five Perl programs (HaploFind.pl, GeneConversionFind.pl, BGC_Calculator.pl, LocalGC_calculator.pl, AT_vs_GC.pl, RandomGC_Calculator.pl). Detailed description and scripts of all our Perl programs, their instruction manuals, the command lines for execution of programs, and examples of output files can be found in the Additional file 1.

Haplotypes for every chromosomal segment were computationally constructed from 50 adjacent frequent genetic variants (MFA > 25\%). Since more than $90 \%$ of these frequent genetic variants are presented by SNPs, we call these variants as SNPs for simplicity throughout the text. However, for putative BGC events having "flip-over" alleles inside common haplotypes we considered only SNPs (all indels were rejected from consideration).

Our computer characterization of common haplotypes has been elaborated in the predecessor project [35]. In that research we tried different parameters for the threshold for frequent SNPs (Minor allele frequency (MAF) $10 \%, 20,25$, or $30 \%$ ) and the number of frequent SNPs in the haplotypes $(30,50$, or 70$)$. In the present paper we chose the default parameters of the previous research (MAF $=25 \%$, number of SNPs in the haplotype $=50$ ), which allow identifying an optimal amount of common haplotypes. The number of common haplotypes and the linkage disequilibrium between SNP within a common haplotype varies from one chromosomal segment to another as described in Dutta et al., [35].

The exact of base pair changes from Common haplotype to Acceptor haplotype have been characterized by the Perl script GeneConversionFind.pl. 
Statistical analyses were performed using $R$ package [36]. One sample T-test was used to calculate the standard deviations for the occurrence of common haplotypes. The standard errors for the 'AT $\rightarrow \mathrm{GC}$ ' and 'GC $\rightarrow$ AT' events were calculated with the formula using the Rule of Sample Proportions

$$
\mathbf{S E}=\sqrt{\frac{\mathbf{p}(\mathbf{1 - p})}{N}}
$$

Where $\mathrm{N}$ is total number of gene conversion events $(\mathrm{AT} \rightarrow \mathrm{GC}$ and GC $\rightarrow \mathrm{AT}$ ), $\mathrm{p}$ is the proportion of AT $\rightarrow$ $\mathrm{GC}$ events and $(1-\mathrm{p})$ is the proportion of GC $\rightarrow \mathrm{AT}$ events.

All our programs are freely available from our website (http://bpg.utoledo.edu/ afedorov/lab/BGC.html) [37]. The entire dataset of all haplotypes for each 56,328 chromosomal segments generated by our programs is also available from this web site (this dataset is too big to place it in Additional file 1).

\section{Results}

Chromosomal segments and common Haplotypes (CHs)

All human autosomes were divided into 56,328 consecutive segments with a default size of $500 \mathrm{~kb}$ as illustrated in Fig. 1a. A complete list of segments for all chromosomes is presented in Table 1. For each chromosomal segment, we determined SNP haplotypes built from 50 adjacent genetic variants occurring with high frequency (Minor Allele Frequency > 25\%) in 1092 individuals. Each of 1092 individuals from phase 1 of the 1000 Genomes Project is represented by two haplotypes that correspond to the two parents of the sequenced individual. All 2184 haplotypes were ranked by their occurrences as explained in Fig. 1b. When a haplotype was found 100 or more times among 1092 studied individuals, it was considered a Common Haplotype $(\mathrm{CH})$. The program HaploFind.pl automatically lists all common and rare haplotypes among all autosomes in 56,328 segments. The average size of our haplotypes $(47.8 \mathrm{~kb})$ is congruent with the findings of Gabriel and co-authors

Table 1 Distribution of computational segments and putative gene conversion events in all autosomes

\begin{tabular}{|c|c|c|c|c|c|c|c|c|c|}
\hline \multirow{2}{*}{$\begin{array}{l}\text { Chromo- } \\
\text { some }\end{array}$} & \multirow{2}{*}{$\begin{array}{l}\text { \# of } \\
\text { Segments }\end{array}$} & \multicolumn{4}{|c|}{ Rare haplotype count = 1} & \multicolumn{4}{|c|}{ Rare haplotype count $<=5$} \\
\hline & & $\begin{array}{l}\mathrm{AT} \rightarrow \mathrm{GC} \\
\text { cases }\end{array}$ & $\begin{array}{l}\mathrm{GC} \rightarrow \mathrm{AT} \\
\text { cases }\end{array}$ & $\begin{array}{l}\text { No Base } \\
\text { Change cases }\end{array}$ & $\begin{array}{l}\text { Total } \\
\text { cases }\end{array}$ & $\begin{array}{l}\text { AT } \rightarrow \text { GC } \\
\text { cases }\end{array}$ & $\begin{array}{l}\mathrm{GC} \rightarrow \mathrm{AT} \\
\text { cases }\end{array}$ & $\begin{array}{l}\text { No Base } \\
\text { Change cases }\end{array}$ & $\begin{array}{l}\text { Total } \\
\text { cases }\end{array}$ \\
\hline 1 & 4359 & 1964 & 1677 & 637 & 4278 & 8973 & 8200 & 3181 & 20,354 \\
\hline 2 & 4660 & 2318 & 1921 & 760 & 4999 & 10,514 & 8928 & 3605 & 23,047 \\
\hline 3 & 4048 & 1977 & 1640 & 632 & 4249 & 8785 & 7715 & 3196 & 19,696 \\
\hline 4 & 4191 & 1945 & 1891 & 722 & 4558 & 9034 & 8442 & 3373 & 20,849 \\
\hline 5 & 3687 & 1786 & 1513 & 629 & 3928 & 7842 & 7190 & 3052 & 18,084 \\
\hline 6 & 3869 & 1791 & 1614 & 629 & 4034 & 8516 & 7835 & 2975 & 19,326 \\
\hline 7 & 2838 & 1361 & 1227 & 492 & 3080 & 6133 & 5431 & 2311 & 13,875 \\
\hline 8 & 3179 & 1631 & 1287 & 560 & 3478 & 7124 & 6170 & 2691 & 15,985 \\
\hline 9 & 2389 & 1163 & 963 & 420 & 2546 & 4898 & 4253 & 1814 & 10,965 \\
\hline 10 & 2830 & 1311 & 1112 & 423 & 2846 & 5897 & 5283 & 1997 & 13,177 \\
\hline 11 & 2838 & 1352 & 1196 & 479 & 3027 & 5943 & 5444 & 2302 & 13,689 \\
\hline 12 & 2676 & 1150 & 1035 & 391 & 2576 & 5475 & 4978 & 1953 & 12,406 \\
\hline 13 & 2135 & 948 & 772 & 282 & 2002 & 4334 & 4000 & 1518 & 9852 \\
\hline 14 & 1852 & 862 & 757 & 277 & 1896 & 3781 & 3368 & 1422 & 8571 \\
\hline 15 & 1647 & 789 & 627 & 251 & 1667 & 3362 & 2822 & 1176 & 7360 \\
\hline 16 & 1723 & 794 & 629 & 367 & 1790 & 3440 & 2787 & 1501 & 7728 \\
\hline 17 & 1540 & 713 & 606 & 201 & 1520 & 3144 & 2709 & 1027 & 6880 \\
\hline 18 & 1629 & 720 & 603 & 226 & 1549 & 3057 & 2728 & 1110 & 6895 \\
\hline 19 & 1334 & 662 & 511 & 196 & 1369 & 2678 & 2314 & 868 & 5860 \\
\hline 20 & 1272 & 559 & 449 & 146 & 1154 & 2350 & 2083 & 785 & 5218 \\
\hline 21 & 840 & 384 & 326 & 121 & 831 & 1620 & 1411 & 574 & 3605 \\
\hline 22 & 792 & 403 & 290 & 125 & 818 & 1643 & 1321 & 523 & 3487 \\
\hline
\end{tabular}

The first two columns of Table 1 lists the number of computationally generated segments in different human autosomes. The next four columns describe number of AT to GC, number of GC to AT, number of 'No Base Change' and total mismatch repair cases respectively in all autosomes when only single rare haplotype occurrence in the 1092 genomes was considered. The last four columns present number of AT to GC cases, number of GC to AT cases, number of 'No Base Change' cases and total mismatch repair cases respectively in all autosomes when rare haplotype occurrence $<=5$ in the 1092 genomes was considered 
[38]. They found that most of the human genome can be divided into blocks/segments of substantial size and, within each of them, very few common haplotypes capture a vast majority $(\sim 90 \%)$ of the chromosomes in each population.

\section{Putative gene conversion events}

To test the gBGC hypothesis, we identified the putative cases of nucleotide changes (SNP flip-overs) due to gene conversion events as illustrated in Fig. 1b using Perl script GeneConversionFind.pl. The script GeneConversionFind.pl identifies individuals who have a common haplotype inherited from one parent and another almost identical (49 matching alleles out of 50) but rare haplotype (occurrence $\leq 5$ times among 1092 individuals) inherited from another parent. In the rare haplotype, the only site, which is not identical to the common haplotype, has a SNP flipover (replacement of an allele inherited from one parent by its complementary allele inherited from another parent). We named this polymorphic site an 'Acceptor site', and named the rare haplotype an 'Acceptor haplotype'. Such cases of SNP flip-overs represent putative gene conversion events, which may have occurred in the genomes of parents of the analyzed individuals or in their genetic predecessors. We have considered only those cases where the 'Acceptor' haplotype occurs once, twice, and $\leq 5$ times in the 1092 Genomes population. For each case, the program HaploFind.pl identifies the Acceptor site, notes its location in the Acceptor haplotype, reference allele and alternative allele at the Acceptor site, and also computes the type of putative gene conversion event at that particular site. A portion of a representative output file generated by the program is shown at the bottom (right side) in Fig. 1b.
The next Perl script $A T_{-} v s_{-} G C . p l$ computes occurrence of different types of SNP flip-overs (AT- $>$ GC or GC- > AT). The program BGC_Calculator.pl was used to combine and summarize results for different chromosomes together and to generate final results, which are shown in Fig. 2.

When we considered cases where Acceptor Haplotype occurs only once in a person from the 1092 Genomes populations, we found 26,583 AT-> GC SNP flip-overs and noticeably less $(22,646)$ GC- > AT SNP flip-overs. A two-tailed Chi-square test was performed with these numbers and gave us a $p$-value $<10^{-16}$, which is statistically significant. When we registered Acceptor haplotype only once, it may be interpreted as sequencing error. However, occurrence of the same Acceptor haplotype in more than one individual reduces the possibilities of such false positives. Therefore, in addition, we identified all SNP flip-over cases where the same acceptor haplotype was found in 2 to 5 individuals (see Fig. 2). We observed similar trends with varying Acceptor haplotype occurrences. This fact testifies that sequencing errors cannot be the reason for our observations.

When the Acceptor Haplotypes which occurred two times among the 1092 individuals were considered, 34,497 and 32,190 cases of AT-> GC and GC->AT conversion events were found respectively. When Acceptor Haplotypes which occurred in less or equal to five persons were considered, 118,543 AT- > GC conversions were identified compared to 105,412 cases of GC- > AT conversions. Chi-square tests performed in each case above resulted in a p-value $<10^{-16}$, which shows the statistical significance of the results. All in all, we report a $11.7 \%$ bias in $\mathrm{AT}->\mathrm{GC}$ over the GC-> AT SNP flipovers.

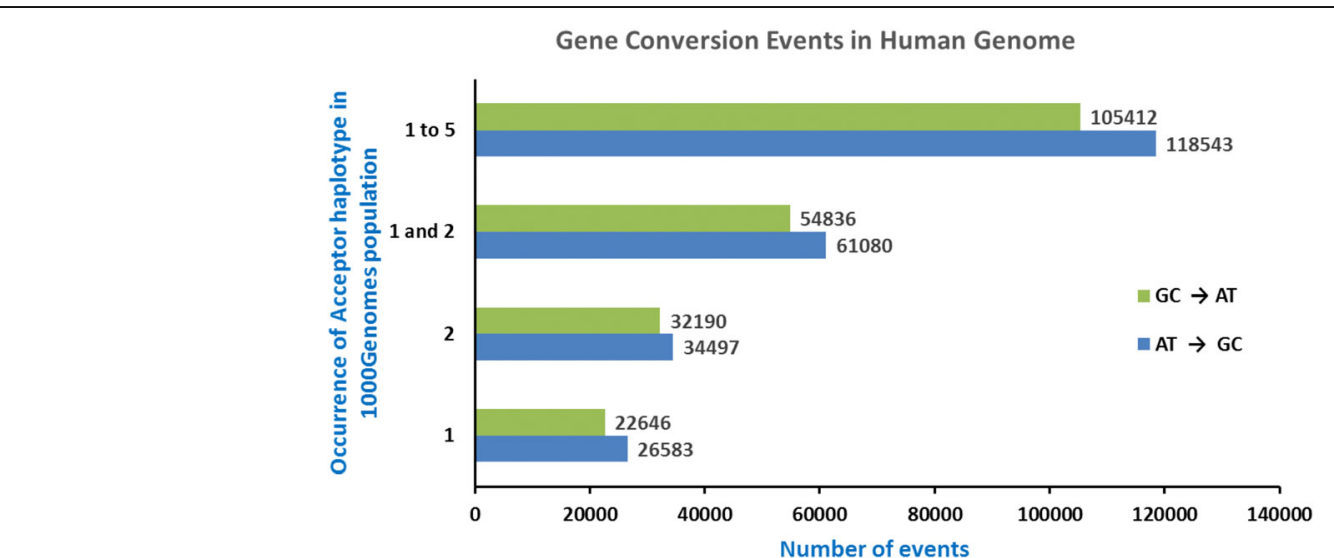

Fig. 2 Number of AT - > GC vS GC - > AT changes due to putative base pair conversion events. The number of identified base pair conversion events is presented along the horizontal axis, while the vertical axis shows the different computational conditions for registration of these events. We considered cases where the rare haplotype (with the Acceptor site) occurs only once among the 1092 individuals (labeled as 1, at the bottom), twice among the 1092 individuals (labeled as 2), single and double occurrences taken together (labeled as 1 and 2) and less than or equal to 5 occurrences among the 1092 individuals (labeled as 1 to 5) 


\section{Local GC content}

Distribution of GC-content at the sites of the conversion events is an important consideration in the BGC hypothesis. Therefore, we calculated local GC content within 100 nucleotide-long window considering 50 nucleotides before and after each putative AT - > GC or GC - > AT conversion site. Our laboratory had previously developed an approach to evaluate various local biases in nucleotide composition and intensively studied non-randomness of such local nucleotide compositions in the human genome $[39,40]$. In these examinations 100 nucleotide-long scanning window had been chosen as a default parameter in these genomic sequence analyses. Thus, we keep the same window size in this project. We used the program LocalGC_calculator.pl to calculate local GC content around 22,646 AT-> GC conversion events and same number of GC->AT sites. Another program RandomGC_Calculator.pl was used to calculate local GCcontent around 22,646 randomly selected sites, which served as a control for the overall distribution of GC-rich regions. These results are presented in Fig. 3. It is wellestablished that recombination is more frequent in GCrich regions [15], and consequently BGC events should occur more frequently in GC-rich regions of the genome. The distribution of local GC-content around putative BGC sites obtained from our computations confirms this conjecture. Indeed the rate of both AT- > GC and GC- > AT SNP flip-overs in GC-rich regions is about $22 \%$ more frequent than the random expectation. We performed Chi-squared test of Goodness-of-Fit with the null hypothesis that there is no difference between the expected distribution of GC content (distribution around random sites) and the observed distributions (distributions of Local GC content around the AT- $>$ GC and GC- $>$ AT sites). The $p$-value for the test comparing AT- $>\mathrm{GC}$ and random distributions was $<2.2 \times 10^{-16}$. The test comparing GC- $>$ AT and random distributions also resulted in a $\mathrm{p}$-value $<2.2 \times 10^{-16}$. These extremely low $p$-values show that the null hypothesis is not true and both the observed distributions of GC content are significantly different than the distribution of GC content around random sites. We did not see a significant difference between the distribution of local GC-content for AT- > GC events versus GC$>$ AT events. Our results confirm that, in AT-rich regions (GC-composition $<40 \%$ ), there are $\sim 10 \%$ fewer events of gBGC base pair flip-over compared to random expectation (blue and red lines are $~ 10 \%$ lower than yellow line on the left side of Fig. 3). In contrast, in GC-rich regions (GC composition $>44 \%$ ), there are $\sim 25 \%$ more putative gBGC base changes over the random expectation (blue and red lines are higher than yellow line on the right side of Fig. 3). These results are in line with gBGC theory. However, the detected disparity is only moderate (10-25\% difference from random distribution on Fig. 3).

\section{Discussion}

What is the average number of SNP flip-overs in a human gamete? The estimation of this number is essential because SNP flip-overs change SNP-haplotypes and linkage disequilibrium between SNPs. This effect should be taken into consideration in various programs used for deciphering phenotypes from SNP patterns because noticeable SNP flip-over process constantly modifies these patterns and reduces linkage disequilibrium between neighboring SNPs. SNP flip-over occurs during NCO meiotic recombination events when one allele is replaced by its counterpart allele, while the neighboring SNPs in the haplotype remain the same. NCOs are up to 15 times more frequent than COs [41-45]. A recent study estimated $228 \mathrm{NCO}$ events on average per generation in humans [27]. At the same time, average length of $\mathrm{NCO}$ heteroduplex tracts are much shorter than $\mathrm{CO}$ tracts with average $\mathrm{NCO}$ tract length of 75 bp according to several recent studies [27, 44, 46]. Therefore, we estimate $228 \times 75 \mathrm{bp}=17.1 \mathrm{~kb}$ of total NCO heteroduplex length per gamete. On the other hand, the number of heterozygous sites for Europeans and Asian individuals in the 1000 genomes dataset are about 2.3 million, and about 3.3 million for African individuals [34]. Taking these groups together, on average there is about one heterozygous site per $1.2 \mathrm{~kb}$ in the human genome. Considering all the above, in human meiosis, about 14.2 mismatches $(17.1 \mathrm{~kb} / 1.2 \mathrm{~kb})$ should be formed within all NCO heteroduplexes of a gamete. During repair, only half of these 14.2 mismatches should resolve into SNP flip-overs, while in the other half of cases, MMR should restore the original alleles within original haplotypes. This leaves 7.1 SNP flip-overs per gamete. They represent up to a quarter of all new mutations in a gamete (there are from 20 to 50 novel mutations in a gamete according to different estimations [47-49]). Hence, SNP flip-over has a substantial impact on nucleotide changes and should be considered in any SNP dynamics analyses.

The second important question we address is the number of AT $\rightarrow$ GC versus GC $\rightarrow$ AT base-pair conversions per human gamete due to gBGC. To estimate this number, we should consider both $\mathrm{CO}$ and $\mathrm{NCO}$ cases since both results in base pair conversions. The estimated sex-averaged number of COs per generation is $\sim 30$ [50]. We will use the average $\mathrm{CO}$ heteroduplex tract length of $600 \mathrm{bp}$ for humans, which is consistent with several current studies $[46,51]$. So, we estimate in total $30 \times 600 \mathrm{bp}=18 \mathrm{~kb}$ of $\mathrm{CO}$ heteroduplex tracts length per gamete. Thus, number of mismatches formed in all CO heteroduplexes during human meiosis is about $15(18 \mathrm{~kb} / 1.2 \mathrm{~kb})$. In the previous paragraph we already calculated that the average number of mismatches in NCO heteroduplexes is 14.2 per gamete. Since, half of mismatches should be resolved in base-pair changes, the total number of base-pair changes due to both $\mathrm{NCO}$ and $\mathrm{CO}$ will be, on average, 14.6 events per gamete. According 


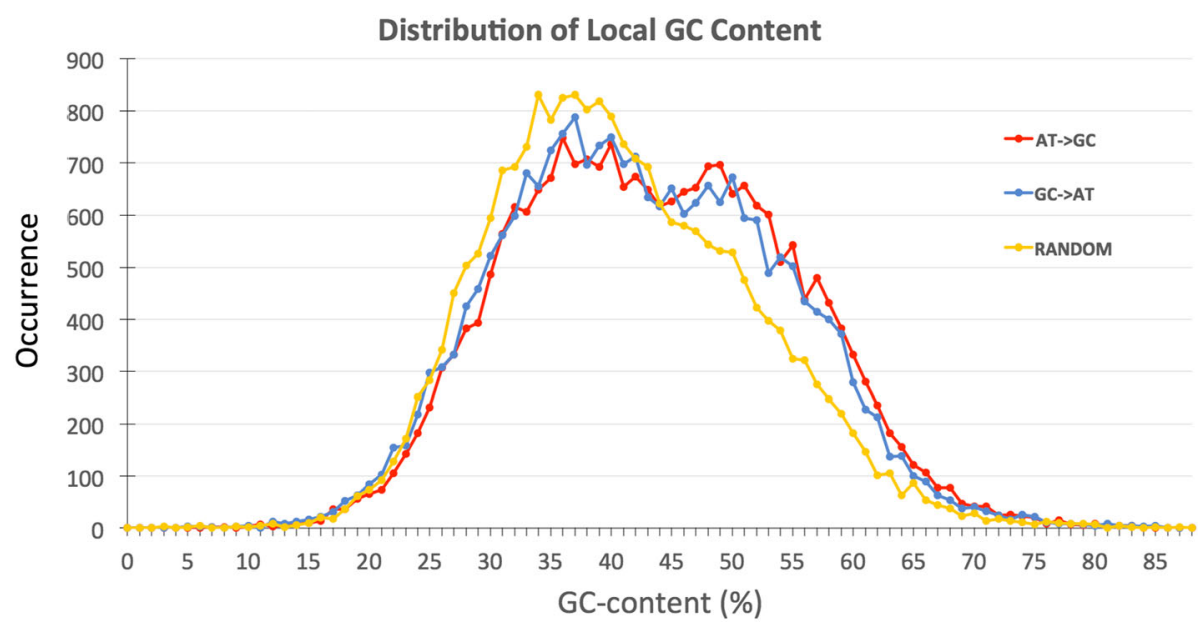

Fig. 3 Distribution of local GC content in regions surrounding AT - > GC vs GC - > AT conversion events. Local GC content was calculated within 100 bp window by considering 50 nucleotides before and after each putative AT - > GC or GC - > AT conversion siteThe red line shows the distribution of local GC content around 22,646 AT - > GC conversion events while the blue line presents the local GC content around the same number of GC - > AT cases. The yellow line (control) represents local GC content around same number of sites selected randomly, independent of gene conversion events.

to our calculations (Table 1), 15.4\% of SNP flip-overs do not cause AT/GC base pair changes, $45.7 \%$ create AT- > GC base pair replacements, while $38.9 \%$ are responsible for the reverse GC- > AT replacements. Thus, on average, 14.6 base-pair change events should generate 2.2 cases with no GC/AT changes (i.e., GC $<->\mathrm{CG}$ and $\mathrm{AT}<->$ TA), 6.7 cases with AT- > GC base pair conversions, and 5.7 cases of GC- > AT conversions. In sum, every human gamete should generate an excess of $1 \mathrm{AT}->\mathrm{GC}$ base pair changes due to gBGC episodes. This number is twice the estimates for yeast genomes [14]. At the same time, our assessment is lower than that of Williams and coauthors who evaluated that $68 \%$ heterozygous AT/GC SNPs transmit GC alleles [27]. The overabundance of 1 AT- > GC base pair changes per gamete, if occurring over millions of years in mammals, may yield significant biases in GCcompositions along chromosomes. However, this effect should be evaluated only in combination with an influx of de novo mutations, which is roughly $20-50$ mutations per gamete [47-49]. Thus, overabundance of 1 AT- $>$ GC base changes per gamete due to gBGC represents only $2-5 \%$ of all new mutations in the gamete genome and might be over-shadowed by other mutational processes. Distribution of novel mutations is uneven along the genome and depends on the local nucleotide composition at the site of mutations. Our laboratory reported a strong fixation bias favoring AT $->\mathrm{GC}$ mutations in GC-rich regions in humans and the opposite fixation bias favoring GC - > AT mutations in AT-rich regions and other fixation biases (e.g. $\mathrm{Pu}->$ Py in pyrimidine rich regions) [40]. Therefore, estimation of the total effect of mutations and conversions on the genomic GC composition is very intricate and still awaits thoughtful modeling.

\section{Conclusions}

During the process of meiotic non-crossover recombination, a human gamete acquires about 7 SNP flip-over events, in which one allele is replaced by its complementary allele while the neighboring SNPs in the haplotype remain the same. On an average, GC-Biased Gene Conversion increments the GC-content by substitution of one AT pair by one GC pair in every haploid human genome. Happening over millions of years of evolution, this smallest bias may be a noticeable force in changing the nucleotide composition landscape along chromosomes.

\section{Additional file}

Additional file 1: Instruction manual for Perl programs for testing BGC hypothesis. This file contains detailed instructions and protocols of Perl programs for construction and analysis of haplotypes of frequent genetic variants. (DOCX $334 \mathrm{~kb})$

\section{Abbreviations}

$\mathrm{CH}$ : Common haplotype; CO: Crossover; gBGC: GC-biased gene conversion; MAF: Minor allele frequency; MMR: DNA Mismatch Repair; NCO: Non- crossover; SNP: Single nucleotide polymorphism

\section{Acknowledgements}

We are grateful to Drs. Robert Blumenthal and Sadik Khuder, University of Toledo Health Science Campus, for their insightful discussions of the project.

\section{Funding}

We appreciate the financial support from the Department of Medicine and School of Biomarker Research and Individualized Medicine, University of Toledo, to conduct our research. This support included stipends for PhD students and also purchasing Linux workstation used for all computations.

Availability of data and materials

The entire dataset supporting the results is available from our web site (http://bpg.utoledo.edu/ afedorov/lab/BGC.html) [37]. In addition, all our programs are also freely available from this website. 
Detailed description and scripts of all our Perl programs, their instruction manuals, the command lines for execution of programs, and examples of output files can be found in the Additional file 1 .

Genotype datasets for all the human chromosomes of the 1092 human genomes were downloaded from (ftp://ftp-trace.ncbi.nih.gov/1000genomes/ $\mathrm{ftp} / \mathrm{release} / 20110521 /$ ) [33].

\section{Authors' contributions}

AF initiated and designed the study and wrote the manuscript. RD developed computer programs for haplotype characterization, conducted computation experiments, performed data analysis, and contributed to writing of the manuscript. LF jointly supervised and designed the study and contributed to writing of the manuscript. ASM, XC, SQ, and JS developed the approach for haplotype analyses, performed data analysis and contributed to creation of Perl scripts. All authors read and approved the final manuscript.

\section{Ethics approval and consent to participate}

Not applicable.

\section{Consent for publication}

Not applicable.

\section{Competing interests}

Author Alexei Fedorov is a section editor of BMC Genomics. The authors declare that they have no other competing interest.

\section{Publisher's Note}

Springer Nature remains neutral with regard to jurisdictional claims in published maps and institutional affiliations.

\section{Author details}

${ }^{1}$ Program in Biomedical Sciences, University of Toledo, Health Science Campus, Toledo, $\mathrm{OH} 43614$, USA. ${ }^{2}$ Department of Medicine, University of Toledo, Health Science Campus, Toledo, OH 43614, USA. ${ }^{3}$ Program in Bioinformatics and Proteomics/Genomics, University of Toledo, Health Science Campus, Toledo, $\mathrm{OH} 43614$, USA. ${ }^{4}$ SURF Program, University of Toledo, Health Science Campus, Toledo, OH 43614, USA. ${ }^{5}$ GEMA-biomics, Ottawa Hills, Lucas County, OH 43606, USA. ${ }^{6}$ Present Address: Center for Cardiovascular and Pulmonary Research, Nationwide Children's Hospital, 700 Children's Dr, Columbus, OH, USA. ${ }^{7}$ Present Address: Biochemistry and Molecular Biology Graduate Program, Cumming School of Medicine, University of Calgary, Calgary, AB T2N4N1, Canada. ${ }^{8}$ College of Arts and Sciences, Washington University in St. Louis, 1 Brookings Dr, St. Louis, MO 63130, USA.

Received: 28 September 2017 Accepted: 12 March 2018

Published online: 16 April 2018

\section{References}

1. Bernardi G. The vertebrate genome: isochores and evolution. Mol Biol Evol. 1993;10(1):186-204.

2. Salinas J, Zerial M, Filipski J, Crepin M, Bernardi G. Nonrandom distribution of MMTV proviral sequences in the mouse genome. Nucleic Acids Res. 1987; 15(7):3009-22.

3. Matassi G, Sharp PM, Gautier C. Chromosomal location effects on gene sequence evolution in mammals. Curr Biol. 1999;9(15):786-91.

4. Wolfe KH, Sharp PM, Li WH. Mutation rates differ among regions of the mammalian genome. Nature. 1989;337(6204):283-5.

5. Cuny G, Soriano P, Macaya G, Bernardi G. The major components of the mouse and human genomes. 1. Preparation, basic properties and compositional heterogeneity. Eur J Biochem. 1981;115(2):227-33.

6. Duret L, Eyre-Walker A, Galtier N. A new perspective on isochore evolution. Gene. 2006:385:71-4

7. Holmquist GP. Chromosome bands, their chromatin flavors, and their functional features. Am J Hum Genet. 1992;51(1):17-37.

8. Eyre-Walker A. Recombination and mammalian genome evolution. Proc Biol Sci. 1993:252(1335):237-43.

9. Eyre-Walker A. Evidence of selection on silent site base composition in mammals: potential implications for the evolution of isochores and junk DNA. Genetics. 1999;152(2):675-83.
10. Galtier N, Piganeau G, Mouchiroud D, Duret L. GC-content evolution in mammalian genomes: the biased gene conversion hypothesis. Genetics. 2001;159(2):907-11.

11. Galtier N, Duret L, Glemin S, Ranwez V. GC-biased gene conversion promotes the fixation of deleterious amino acid changes in primates. Trends Genet. 2009;25(1):1-5.

12. Blain JF, Aumont N, Theroux L, Dea D, Poirier J. A polymorphism in lipoprotein lipase affects the severity of Alzheimer's disease pathophysiology. Eur J Neurosci. 2006;24(5):1245-51.

13. Surtees JA, Argueso JL, Alani E. Mismatch repair proteins: key regulators of genetic recombination. Cytogenet Genome Res. 2004;107(3-4):146-59.

14. Lesecque Y, Mouchiroud D, Duret L. GC-biased gene conversion in yeast is specifically associated with crossovers: molecular mechanisms and evolutionary significance. Mol Biol Evol. 2013;30(6):1409-19.

15. Meunier J, Duret L. Recombination drives the evolution of GC-content in the human genome. Mol Biol Evol. 2004;21(6):984-90.

16. Necsulea A, Popa A, Cooper DN, Stenson PD, Mouchiroud D, Gautier C, Duret L. Meiotic recombination favors the spreading of deleterious mutations in human populations. Hum Mutat. 2010;32(2):198-206.

17. Pessia E, Popa A, Mousset S, Rezvoy C, Duret L, Marais GA. Evidence for widespread GC-biased gene conversion in eukaryotes. Genome Biol Evol. 2012;4(7):675-82

18. Harrison RJ, Charlesworth B. Biased gene conversion affects patterns of codon usage and amino acid usage in the Saccharomyces sensu stricto group of yeasts. Mol Biol Evol. 2010;28(1):117-29.

19. Yang S, Yuan Y, Wang L, Li J, Wang W, Liu H, Chen JQ, Hurst LD, Tian D. Great majority of recombination events in Arabidopsis are gene conversion events. Proc Natl Acad Sci U S A. 2012;109(51):20992-7.

20. Kent CF, Minaei S, Harpur BA, Zayed A. Recombination is associated with the evolution of genome structure and worker behavior in honey bees. Proc Natl Acad Sci U S A. 2012;109(44):18012-7.

21. Doring $Y$, Zechner U, Roos C, Rosenkranz D, Zischler H, Herlyn H. Accelerated evolution of Fetuin-a (FETUA, also AHSG) is driven by positive Darwinian selection, not GC-biased gene conversion. Gene. 2010;463(1-2):49-55.

22. Robinson MC, Stone EA, Singh ND. Population genomic analysis reveals no evidence for GC-biased gene conversion in Drosophila melanogaster. Mol Biol Evol. 2014;31(2):425-33. https://doi.org/10.1093/molbev/mst220. Epub 2013 Nov 9. PubMed PMID: 24214536.

23. Assis R, Kondrashov AS. Nonallelic gene conversion is not GC-biased in Drosophila or primates. Mol Biol Evol. 2011;29(5):1291-5.

24. Mugal CF, Arndt PF, Ellegren H. Twisted signatures of GC-biased gene conversion embedded in an evolutionary stable Karyotype. Mol Biol Evol. 2013;30(7):1700-12.

25. Katzman S, Capra JA, Haussler D, Pollard KS. Ongoing GC-biased evolution is widespread in the human genome and enriched near recombination hot spots. Genome Biol Evol. 2011;3:614-26.

26. Pollard KS, Salama SR, King B, Kern AD, Dreszer T, Katzman S, Siepel A, Pedersen JS, Bejerano $G$, Baertsch $R$, et al. Forces shaping the fastest evolving regions in the human genome. PLoS Genet. 2006;2(10):e168.

27. Williams AL, Genovese G, Dyer T, Altemose N, Truax K, Jun G, Patterson N, Myers SR, Curran JE, Duggirala R, et al. Non-crossover gene conversions show strong GC bias and unexpected clustering in humans. elife. 2015;4.

28. Halldorsson BV, Hardarson MT, Kehr B, Styrkarsdottir U, Gylfason A, Thorleifsson G, Zink F, Jonasdottir A, Jonasdottir A, Sulem $P$, et al. The rate of meiotic gene conversion varies by sex and age. Nat Genet. 2016;48(11):1377-84

29. Durand EY, Eriksson N, McLean CY. Reducing pervasive false-positive identical-by-descent segments detected by large-scale pedigree analysis. Mol Biol Evol. 2014:31(8):2212-22.

30. Li H, Glusman G, Hu H, Shankaracharya, Caballero J, Hubley R, Witherspoon D, Guthery SL, Mauldin DE, Jorde LB, et al. Relationship estimation from whole-genome sequence data. PLoS Genet. 2014;10(1):e1004144.

31. Huff CD, Witherspoon DJ, Simonson TS, Xing J, Watkins WS, Zhang Y, Tuohy TM, Neklason DW, Burt RW, Guthery SL, et al. Maximum-likelihood estimation of recent shared ancestry (ERSA). Genome Res. 2011;21(5):768-74.

32. Glemin S, Arndt PF, Messer PW, Petrov D, Galtier N, Duret L. Quantification of GC-biased gene conversion in the human genome. Genome Res. 2015; 25(8):1215-28.

33. NCBI trace FTP site [ftp://ftp-trace.ncbi.nih.gov/1000genomes/ftp/release/ 20110521/. Accessed 4 Mar 2018

34. Genomes Project C, Abecasis GR, Auton A, Brooks LD, DePristo MA, Durbin RM, Handsaker RE, Kang HM, Marth GT, McVean GA. An integrated map of genetic variation from 1,092 human genomes. Nature. 2012;491(7422):56-65. 
35. Dutta R, Mainsah J, Yatskiv Y, Chakrabortty S, Brennan P, Khuder B, Qiu S, Fedorova L, Fedorov A. Intricacies in arrangement of SNP haplotypes suggest "great admixture" that created modern humans. BMC Genomics. 2017:18(1):433.

36. R Development Core Team: $\mathrm{R}$ : a language and environment for statistical computing. In. Vienna, Austria: R foundation for statistical computing; 2010.

37. Downloading web site for 1000 Human Genomes carry widespread signatures of GC biased gene conversion [http://bpg.utoledo.edu/ afedorov/lab/BGC.html ]. Accessed 4 Mar 2018.

38. Gabriel SB, Schaffner SF, Nguyen H, Moore JM, Roy J, Blumenstiel B, Higgins J, DeFelice M, Lochner A, Faggart M, et al. The structure of haplotype blocks in the human genome. Science. 2002;296(5576):2225-9.

39. Bechtel JM, Wittenschlaeger T, Dwyer T, Song J, Arunachalam S, Ramakrishnan SK, Shepard S, Fedorov A. Genomic mid-range inhomogeneity correlates with an abundance of RNA secondary structures. BMC Genomics. 2008;9:284.

40. Prakash A, Shepard SS, He J, Hart B, Chen M, Amarachintha SP, MileyevaBiebesheimer O, Bechtel J, Fedorov A. Evolution of genomic sequence inhomogeneity at mid-range scales. BMC Genomics. 2009;10:513.

41. Odenthal-Hesse L, Berg IL, Veselis A, Jeffreys AJ, May CA. Transmission distortion affecting human noncrossover but not crossover recombination: a hidden source of meiotic drive. PLoS Genet. 2014;10(2):e1004106.

42. Ardlie K, Liu-Cordero SN, Eberle MA, Daly M, Barrett J, Winchester E, Lander ES, Kruglyak L. Lower-than-expected linkage disequilibrium between tightly linked markers in humans suggests a role for gene conversion. Am J Hum Genet. 2001;69(3):582-9.

43. Frisse L, Hudson RR, Bartoszewicz A, Wall JD, Donfack J, Di Rienzo A. Gene conversion and different population histories may explain the contrast between polymorphism and linkage disequilibrium levels. Am J Hum Genet. 2001;69(4):831-43

44. Jeffreys AJ, May CA. Intense and highly localized gene conversion activity in human meiotic crossover hot spots. Nat Genet. 2004;36(2):151-6.

45. Gay J, Myers S, McVean G. Estimating meiotic gene conversion rates from population genetic data. Genetics. 2007;177(2):881-94.

46. Cole F, Baudat F, Grey C, Keeney S, de Massy B, Jasin M. Mouse tetrad analysis provides insights into recombination mechanisms and hotspot evolutionary dynamics. Nat Genet. 2014:46(10):1072-80

47. Conrad DF, Keebler JE, DePristo MA, Lindsay SJ, Zhang Y, Casals F, Idaghdour Y, Hartl CL, Torroja C, Garimella KV, et al. Variation in genome-wide mutation rates within and between human families. Nat Genet. 2011;43(7):712-4.

48. Li H, Durbin R. Inference of human population history from individual whole-genome sequences. Nature. 2011;475(7357):493-6.

49. Kondrashov AS, Shabalina SA. Classification of common conserved sequences in mammalian intergenic regions. Hum Mol Genet. 2002;11 (6):669-74.

50. Fledel-Alon A, Leffler EM, Guan Y, Stephens M, Coop G, Przeworski M. Variation in human recombination rates and its genetic determinants. PLoS One. 2011;6(6)::20321.

51. Capra JA, Hubisz MJ, Kostka D, Pollard KS, Siepel A. A model-based analysis of GC-biased gene conversion in the human and chimpanzee genomes. PLoS Genet. 2013:9(8):e1003684.

\section{Submit your next manuscript to BioMed Central and we will help you at every step:}

- We accept pre-submission inquiries

- Our selector tool helps you to find the most relevant journal

- We provide round the clock customer support

- Convenient online submission

- Thorough peer review

- Inclusion in PubMed and all major indexing services

- Maximum visibility for your research

Submit your manuscript at www.biomedcentral.com/submit

) Biomed Central 